\title{
Digital Clubbing
}

Tatiana Denega MD, Suzanne Alkul BS, Raed Alalawi MD

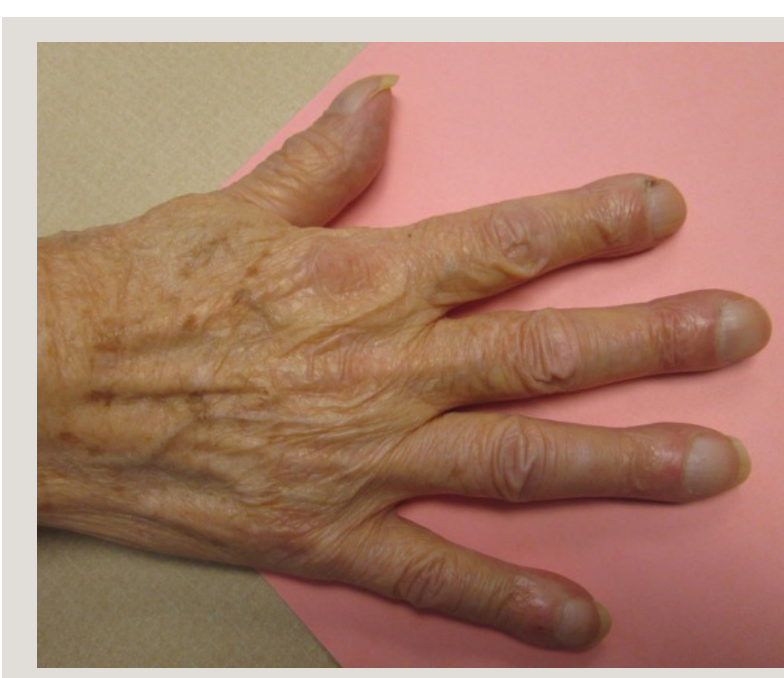

Figure 1 Top view of the right hand.

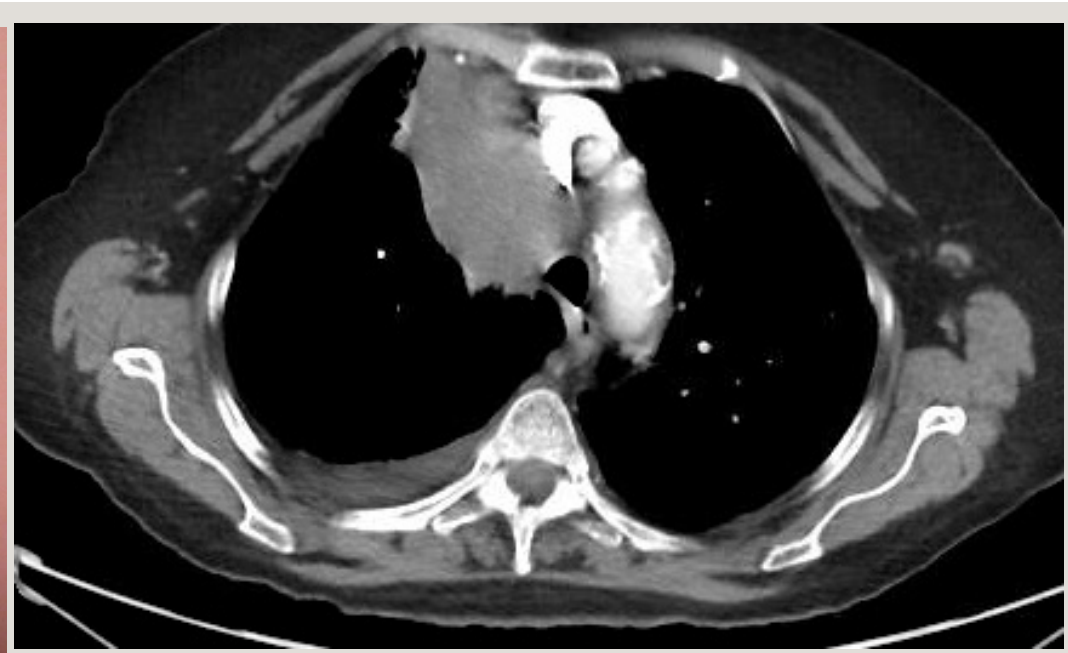

Figure 2 Right upper lobe mass extending into the mediastinum.

\section{CASE}

A 76-year-old woman was admitted to the hospital with progressive shortness of breath. Her symptoms worsened in the previous week and were associated with cough with sputum and episodes of fever. The patient had a long history of smoking and chronic obstructive pulmonary disease. Physical examination revealed distinctive changes in the distal phalanges (Figure 1). Laboratory results demonstrated leukocytosis and normocytic anemia. A lobulated mass in the right anterior upper and middle lung and pneumonia in the right lower lobe were found on the chest $x$-ray. These findings were confirmed by the CT scan of the chest, which also revealed a right mediastinal and a

Corresponding author: Raed Alalawi MD Contact Information: raed.alalawi@ttuhsc.edu DOI: $10.12746 /$ swrccc 2015.0309.117 suprahilar mass, extending into the right main stem bronchus, encasing the right upper and middle lower pulmonary arteries, and resulting in collapse of the right upper and middle lobes (Figures 2, 3). Diagnostic bronchoscopy revealed obstruction of the right main stem bronchus by the tumor tissue, and biopsy of the tissue identified large cell carcinoma. Patency of the bronchus was restored by the endobronchial stent placement, and the patient was placed on antibiotic therapy.

\section{Discussion}

Pulmonary neoplasms may be associated with numerous paraneoplastic syndromes. ${ }^{1}$ Hypertrophic osteoarthropathy (HOA) is a paraneoplastic rheumatologic syndrome, which is observed in $1.87 \%$ patients with lung malignancies. ${ }^{2}$ Its presentation usually involves digital clubbing, also referred to as 


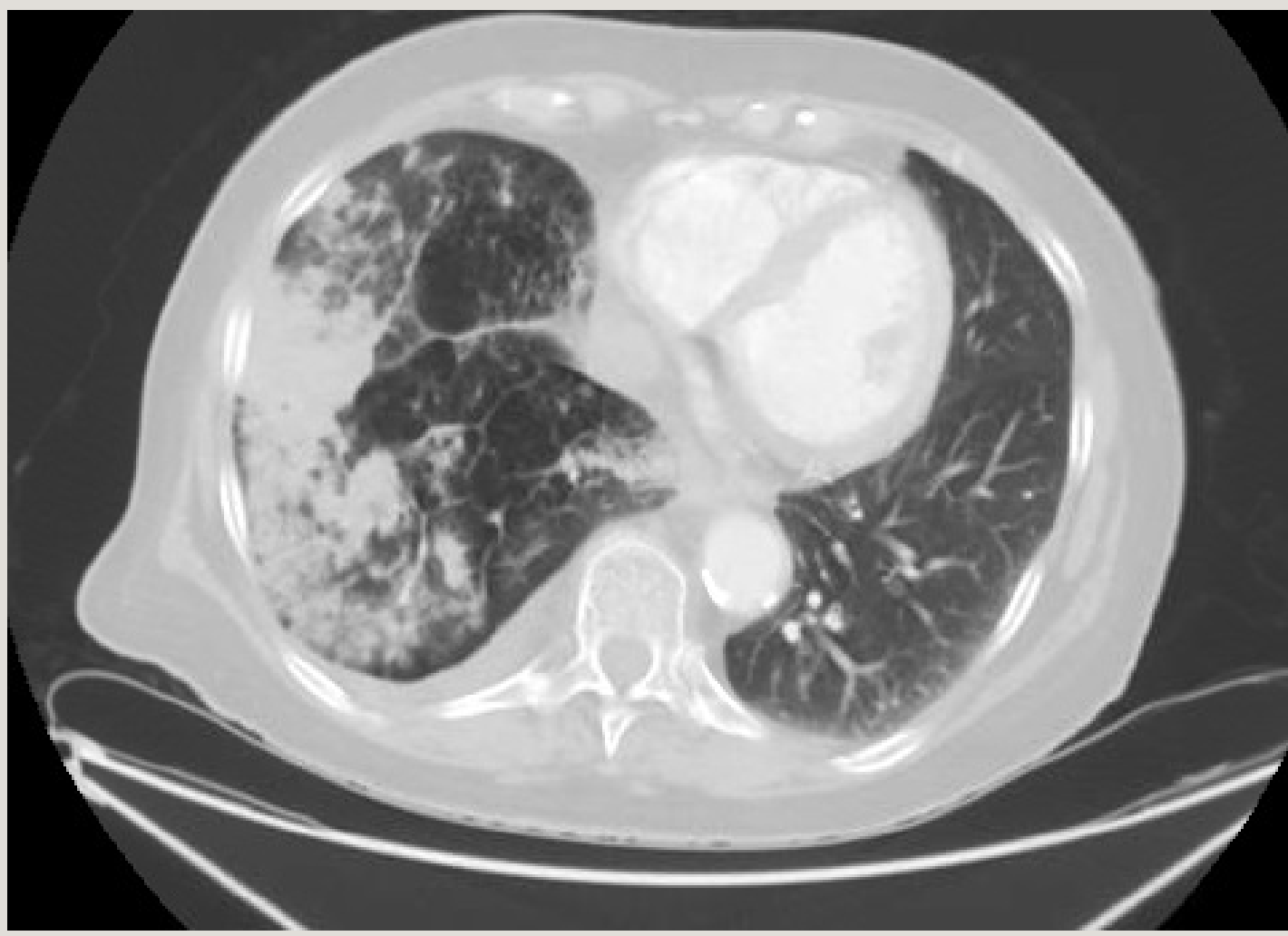

Figure 3 Post-obstructive pneumonia in the right lower lobe.

"drumstick fingers", painful swollen joints, and periosteal new bone formation. ${ }^{10}$ We focus on digital clubbing, as it was present in our patient. Its mechanism of development is attributed to malignant tissue in the lung, which may act as an arteriovenous shunt for megakaryocytes and allow them to bypass the capillary network of the lungs and enter the systemic circulation. ${ }^{3}$ The mechanical hypothesis for the cause of digital clubbing involves the effect of platelet-derived growth factors (PDFGs), which are released during the fragmentation of platelets in the digital capillaries. ${ }^{4}$ PDGF stimulates DNA synthesis in cells of mesenchymal origin (usually the connective tissue-forming cells) and increases vascular permeability. ${ }^{5}$ This increase in vascular connective tissue and collagen deposition leads to an increased distal phalangeal finger depth and hence digital clubbing. Lovibond's sign and Schamroth's sign illustrate the condition as a measurement that is greater than 180 degrees of the angle of nail exiting a proximal nail-fold and the loss of a diamond-shaped profile that is formed by the opposing nail-to-nail index fingers, respectively. ${ }^{6,7}$ A clinical diagnosis is sufficient to determine digital clubbing. However, additional diagnostic studies, such as the bone scintigraphy, are needed to identify the underlying HOA, which usually has symmetrically increased technetium-99m-phosphate uptake in the cortical margins of long bones. Our patient declined additional imaging studies.

The incidence rates of digital clubbing in small (SCLC) and non-small cell (NSCLC) lung cancer vary. Sridhar, et al. reported digital clubbing in $4 \%$ of patients with SCLC and 35\% patients with NSCLC. ${ }^{11}$ Erkan, et al. and Baughman, et al. found similar rates in these two patient groups. ${ }^{8,9}$ Digital clubbing occurs in one-third of patients with lung malignancies. ${ }^{11}$ The absence of digital clubbing does not rule out malignancy, but its presence increases the probability of lung malignancy (likelihood ratio: $3.9 ; 95 \% \mathrm{Cl}: 1.6-9.4){ }^{7}$ 
Author affiliations : Tatiana Denega is a resident in Internal Medicine at Texas tech University Health Sciences Center in Lubbock. Suzanne Alkul is a medical student at TTUHSC in Lubbock. Raed Alalawi is a faculty member in the Department of Internal medicine at TTUHSC in Lubbock.

Submitted: $10 / 6 / 2014$

Accepted: $11 / 20 / 2014$

Reviewers: Zachary Mulkey MD

Conflict of Interest: None

Published electronically: 1/15/2015

\section{References}

1. Kanaji N, Watanabe N, Kita N, Bandoh S, Tadokoro A, Ishii T, Dobashi H, Matsunaga T. Paraneoplastic syndromes associated with lung cancer. World J Clin Oncol 2014; 5(3): 197-223.

2. Qian X, Qin J. Hypertrophic pulmonary osteoarthropathy with primary lung cancer. Oncol Letters 2014; 7(6): 20792082.

3. Dickinson C. The aetiology of clubbing and hypertrophic osteoarthropathy. Europ J Clin Invest 1993; 23(6): 330338.

4. Dickinson C, Martin J. Megakaryocytes and platelet clumps as the cause of finger clubbing. Lancet 1987; 2(8573): 1434-1435.

5. $\quad$ Ross R. Platelet-derived growth factor. Lancet 1989; 1(8648): 1179-1182.

6. Lovibond JL. Diagnosis of clubbed fingers. Lancet 1938; 363-364.

7. Myers KA; Farquhar DRE. Does this patient have clubbing? The rational clinical examination. JAMA 2001; 286(3): 341-347.

8. Baughman RP, Gunther KL, Buchsbaum JA, Lower EE. Prevalence of digital clubbing in bronchogenic carcinoma by a new digital index. Clin Exper Rheum1998; 16(1): 21-6.

9. Erkan ML, Findik S, Kandemir B, Atici AG, Talisöz $\mathrm{H}$. The prevalence of clubbing in different types of lung cancer. Ann Saudi Med 2002; 22(5-6):295-6.

10. Vandemergel X, Renneboog B. Prevalence, aetiologies and significance of clubbing in a department of general internal medicine. Europ J Intern Med 2008; 19(5): 325-329.

11. Sridhar KS, Lobo CF, Altman RD. Digital clubbing and lung cancer. Chest 1998; 114(6):1535-1537. 